\title{
Alcohol Use Disorder and Hepatitis C Prevention and Care in People Who Inject Drugs: The State of Play
}

\author{
Fabienne Marcellin, $\mathrm{PhD}^{1}$ Antoine Jaquet, $\mathrm{MD}, \mathrm{PhD}^{2,3}$ Jeffrey V. Lazarus, $\mathrm{PhD}, \mathrm{MIH}, \mathrm{MA}^{4}$ \\ Patricia Molina, MD, $\mathrm{PhD}^{5}$ Patrizia Carrieri, $\mathrm{PhD}^{1}$
}
${ }^{1}$ Aix Marseille Univ, INSERM, IRD, SESSTIM, Sciences Economiques \& Sociales de la Santé \& Traitement de I'Information Médicale, Marseille, France
2 Univ. Bordeaux, Inserm, Bordeaux Population Health Research Center, UMR 1219, Bordeaux, France
${ }^{3}$ Inserm, ISPED, Bordeaux Population Health Research Center, UMR 1219, Bordeaux, France
${ }^{4}$ Barcelona Institute for Global Health (ISGlobal), Hospital Clínic, University of Barcelona, Barcelona, Spain
${ }^{5}$ Department of Physiology and Alcohol and Drug Abuse Center of Excellence, Louisiana State University Health Sciences Center, New Orleans, Louisiana

Address for correspondence Jeffrey V. Lazarus, PhD, MIH, MA, Barcelona Institute for Global Health (ISGlobal), Hospital Clínic, University of Barcelona, Calle del Rosellón 132, 4th Floor, ES-08036 Barcelona, Spain (e-mail: Jeffrey.Lazarus@isglobal.org).

Semin Liver Dis 2021;41:109-116.

\begin{abstract} Keywords

- alcohol use disorder

- hazardous alcohol consumption

- hepatitis C virus

- people who inject drugs

- treatment

Safe and effective treatment with direct-acting antivirals (DAAs) has ushered in an era in which hepatitis C virus (HCV) elimination, as set out by the World Health Organization, is possible. However, alcohol use disorder (AUD) has the potential to reduce the benefits of prevention interventions and reduce access to and continuity of HCV care in at-risk populations, such as people who inject drugs (PWID). We review the literature on the consequences of AUD on the effectiveness of HCV prevention and the cascade of care in PWID and provide recommendations for future research in the field of alcohol use and HCV.
\end{abstract}

Alcohol use disorder (AUD), defined as a "maladaptive pattern of alcohol use leading to clinically significant impairment or distress," encompasses both alcohol abuse and alcohol dependence. ${ }^{1}$ It can be identified as the presence of at least two criteria from an 11-item list including consumption of alcohol in larger amounts or during longer periods than intended, difficulties in controlling alcohol consumption, and experiencing cravings, withdrawal syndrome, and/or important social and health-related repercussions of alcohol consumption. Globally, AUD is the most prevalent of all substance use disorders, with more than 100 million estimated cases in $2016 .^{2}$

AUD substantially contributes to the burden of liver disease among people with chronic hepatitis $\mathrm{C}$ virus (HCV) infection, notably to the onset of decompensated cirrhosis ${ }^{3}$ and to liver transplantation and mortality. ${ }^{4}$ It is also associated with greater liver damage due to its interactions with immune response, cytotoxicity, and oxidative stress. ${ }^{5}$ People who inject drugs (PWID) are at high risk for AUD, and alcohol use accounts for more than $40 \%$ of liver associated deaths in this population. ${ }^{6}$-Table 1 summarizes the current guidelines for the screening of AUD.

\section{Hepatitis C Elimination: Where Are We Now?}

In 2016, the World Health Organization (WHO) set out the goal of eliminating $\mathrm{HCV}$ as a major public health threat by 2030 , defined as an $80 \%$ reduction in new infections and a $65 \%$ reduction in deaths. ${ }^{7}$ Injecting drug use accounts for $23 \%$ of new HCV infections and one-third of HCV-related deaths. ${ }^{8}$ Current prevention efforts thus center around the population of PWID, ${ }^{9,10}$ which encompasses both individuals actively injecting drugs and those with a history of drug 
Table 1 Screening for alcohol use disorder

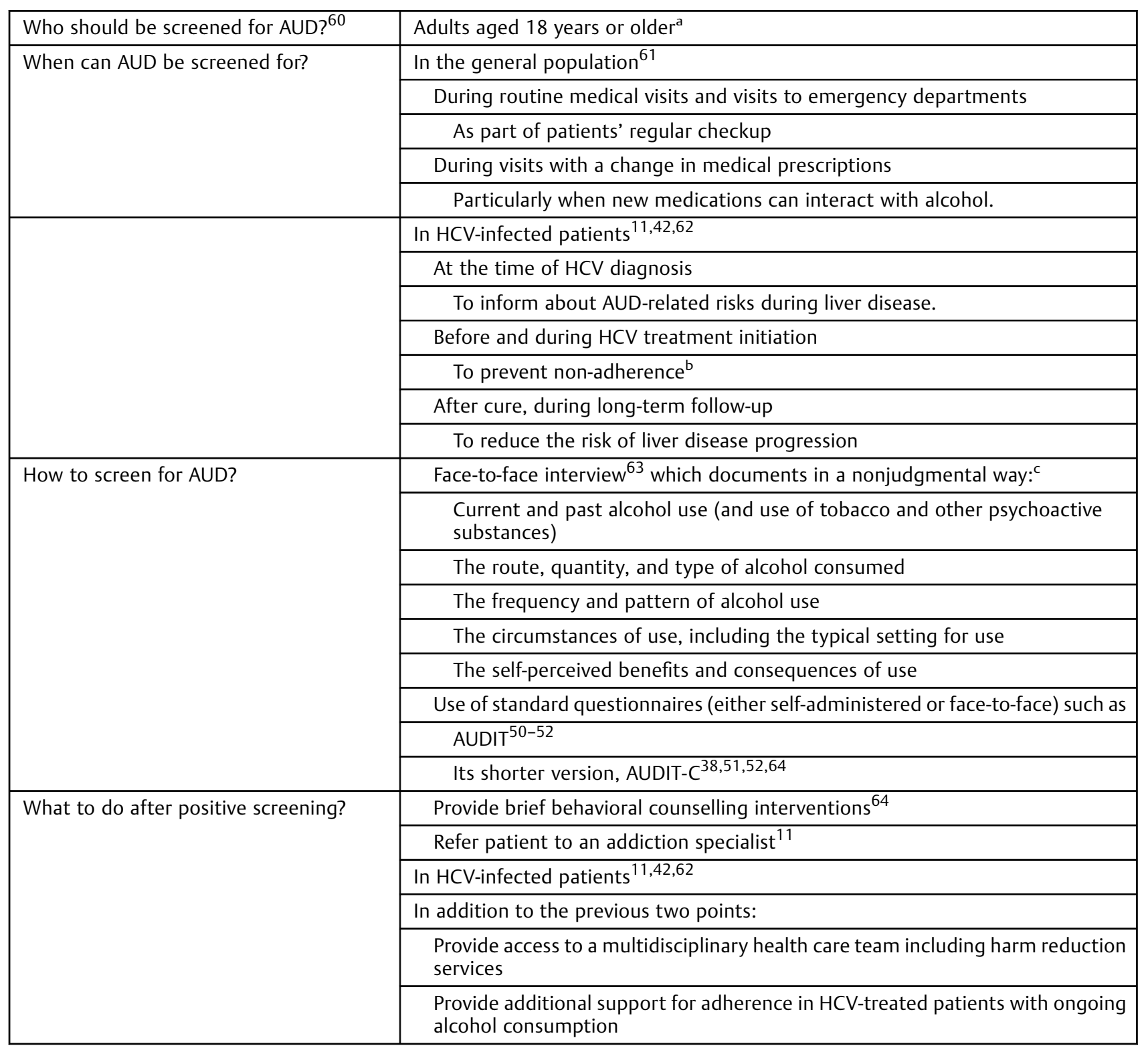

Abbreviations: AUD, alcohol use disorder; AUDIT, Alcohol Use Disorder Identification Test; AUDIT-C, Alcohol Use Disorder Identification Test Consumption; HCV, hepatitis C virus.

${ }^{a}$ AUD screening is especially recommended in certain patient subgroups, such as pregnant women, women planning to conceive, young people, tobacco smokers, or people with health problems, which may be related to alcohol abuse (e.g., high blood pressure, liver disease, depression, and cardiovascular disease). ${ }^{61}$

${ }^{b}$ Ongoing alcohol use does not affect therapeutic outcomes among adherent direct-acting antiviral treated patients. ${ }^{44}$

'Patients' self-report of certain symptoms (e.g., sleep disturbances, gastrointestinal problems, or frequent headaches after the weekend) can facilitate caregivers in opening the discussion about alcohol use. ${ }^{63}$

injection. ${ }^{11}$ However, this population remains largely undiagnosed and untreated, and continues to drive the HCV epidemic.

According to a modelling study addressing the global timing of HCV elimination in 45 high-income countries, ${ }^{12}$ only 9 countries will be able to meet the deadline of the WHO's HCV elimination target by 2030 , whereas 30 are not expected to eliminate HCV before 2050. Key elements to achieve HCV elimination include the development of HCVspecific national strategies and guidelines, the decentralization of HCV screening, universal access to affordable direct- acting antiviral (DAA) treatment, and the implementation of comprehensive HCV-prevention strategies. ${ }^{13}$ Micro-elimination initiatives targeting key populations, such as PWID and subsets of PWID, have the potential to accelerate the HCV global elimination process while being less complex and less costly than full-scale country-level HCV initiatives. ${ }^{14}$ In the case of PWID, these initiatives rely notably on harm reduction services (e.g., drop-in centers, needle and syringe programs [NSPs], opioid agonist therapy [OAT] sites, and outreach services) as they are major entry points for both HCV screening ${ }^{15}$ and "test-and-treat" strategies. ${ }^{8}$ However, 
access to such services remains insufficient globally and must be greatly expanded, as only $1 \%$ of PWID are living in countries with a high coverage of NSP and OAT. ${ }^{16}$ Microelimination among PWID implies scaling up HCV screening to strengthen the initial phases of the HCV continuum of care (i.e., estimating the number of PWID infected, the number of those reached by HCV prevention actions, the percentage of PWID diagnosed, and those aware of their infection) and ensuring that HCV-positive individuals can access appropriate follow-up and care. ${ }^{15}$ This will require multidisciplinary approaches involving, notably, physicians from different specialties, such as addiction and HCV specialists and community-based organizations (peers).

\section{AUD and HCV: Research Needs}

The availability of DAAs, which are safe and highly effective, and the promotion of novel HCV test-and-treat strategies, especially for PWID, have shifted the perspective of research toward exploring interactions between alcohol use and HCV prevention and care. These developments also highlight the need for greater and more innovative research on the impact of alcohol consumption-a modifiable risk factor-on both the effectiveness of prevention interventions and the development or progression of liver disease. ${ }^{17}$ Understanding how, when, and to what extent AUD affects hepatitis prevention interventions is an essential step in identifying and promoting novel approaches to reduce the impact of AUD on risk behaviors. It is also crucial to better document the effect of AUD on HCV treatment uptake and response as they represent two main steps of the $\mathrm{HCV}$ cascade of care. ${ }^{18}$ Despite the existence of review studies focusing on the interactions between alcohol and $\mathrm{HCV}^{19}$ or addressing global barriers to HCV care among PWID, ${ }^{9,20-23}$ there is a paucity of reviews specifically targeting the impact of AUD on HCV prevention and care among PWID.

\section{Study Objectives and Search Strategy}

We present here a narrative review on the effects of AUD on $\mathrm{HCV}$ prevention and care among PWID in the era of HCV elimination. Furthermore, we suggest recommendations to improve the assessment of AUD and to reduce its health impact in this at-risk population. Issues concerning longterm follow-up of HCV-infected PWID after hepatitis C cure have not been explored.

PubMed and the Cochrane library were searched in April 2020 for alcohol use, HCV Medical Subject Headings $(\mathrm{MeSH})$, and free-text terms. We included only articles referring to alcohol use in people at risk of hepatitis $C$ attending NSPs or on OAT (methadone or buprenorphine), people receiving educational interventions for hepatitis $C$ prevention, or people benefiting from hepatitis $\mathrm{C}$ screening and linkage to care. We excluded studies that did not allow for distinguishing between alcohol and drug use and those that did not assess the effect of alcohol use on hepatitis $C$ transmission risk or engagement in care.

\section{Effect of AUD on HCV Prevention among People Who Inject Drugs}

A variety of risk reduction interventions exist to prevent $\mathrm{HCV}$ in PWID, ${ }^{24}$ including NSP and/or OAT, primarily based on methadone or buprenorphine treatment, ${ }^{25}$ peer education interventions, and the recent implementation of test-andtreat strategies. The four primary outcomes of interest when studying the effect of alcohol use on the effectiveness of HCV prevention interventions are (1) seroconversion, (2) persistent injection, (3) sharing of needles, syringes, or other injecting equipment, and (4) unsafe sexual behaviors.

Among PWID, AUD is associated with a higher risk of engaging in unprotected sex and unsafe injecting practices, being HCV-positive, and maintaining injecting practices ${ }^{26-30}$ (-Fig. 1). While the injecting of alcohol itself is rare, it also constitutes a risk behavior in PWID, especially in marginalized individuals. Within this population, alcohol injection is associated with homelessness and the sharing of cookers or filters. $^{31}$

Few studies have specifically investigated the influence of alcohol use on injecting behavior in PWID exposed to NSP. Indeed, in many prevention studies, information about alcohol use is embedded under the term drug and alcohol use, making it nearly impossible to distinguish the impact of AUD from that of drug use. One study that examined 196 PWID in Rhode Island ${ }^{28}$ showed that individuals participating in NSP and who met the criteria for alcohol abuse (according to the third version of the Diagnostic and Statistical Manual of Mental Disorders, DSM-III) had at least a twofold greater risk of needle sharing compared with nondrinkers and moderate alcohol users, even after controlling for other demographic and behavioral factors. A more recent study ${ }^{26}$ of 315 male PWID in rural Puerto Rico attending NSP showed that at-risk alcohol drinking compared with low-risk drinking and no drinking was associated with increased injectingrelated and sexual-related risk behaviors. Another study showed that among 296 male PWID in Nepal, the unavailability of new needles and drinking alcohol were independently associated with sharing injecting equipment among PWID. ${ }^{27}$

In addition to the evidence on the association between alcohol use and injecting-related risk behaviors, evidence suggests that the type of drugs injected may also have an impact on alcohol use. For example, people who inject stimulants have been shown to be significantly more likely to report AUD than people who exclusively inject opiates. ${ }^{32}$ As such, further investigation is required to understand whether the concomitant use of alcohol and specific classes of drugs increases the risk of parenteral transmission of HCV through a higher frequency of unsafe injections.

In high-income countries, AUD is frequent in individuals receiving methadone treatment. ${ }^{33}$ Several studies have shown that AUD increases HCV risk behaviors in individuals receiving OAT. In a U.S. study of 386 individuals entering integrated buprenorphine/naloxone and HIV care, alcohol use during the previous 30 months (irrespective of amount) was associated with an increased risk of unprotected sex. ${ }^{34}$ 


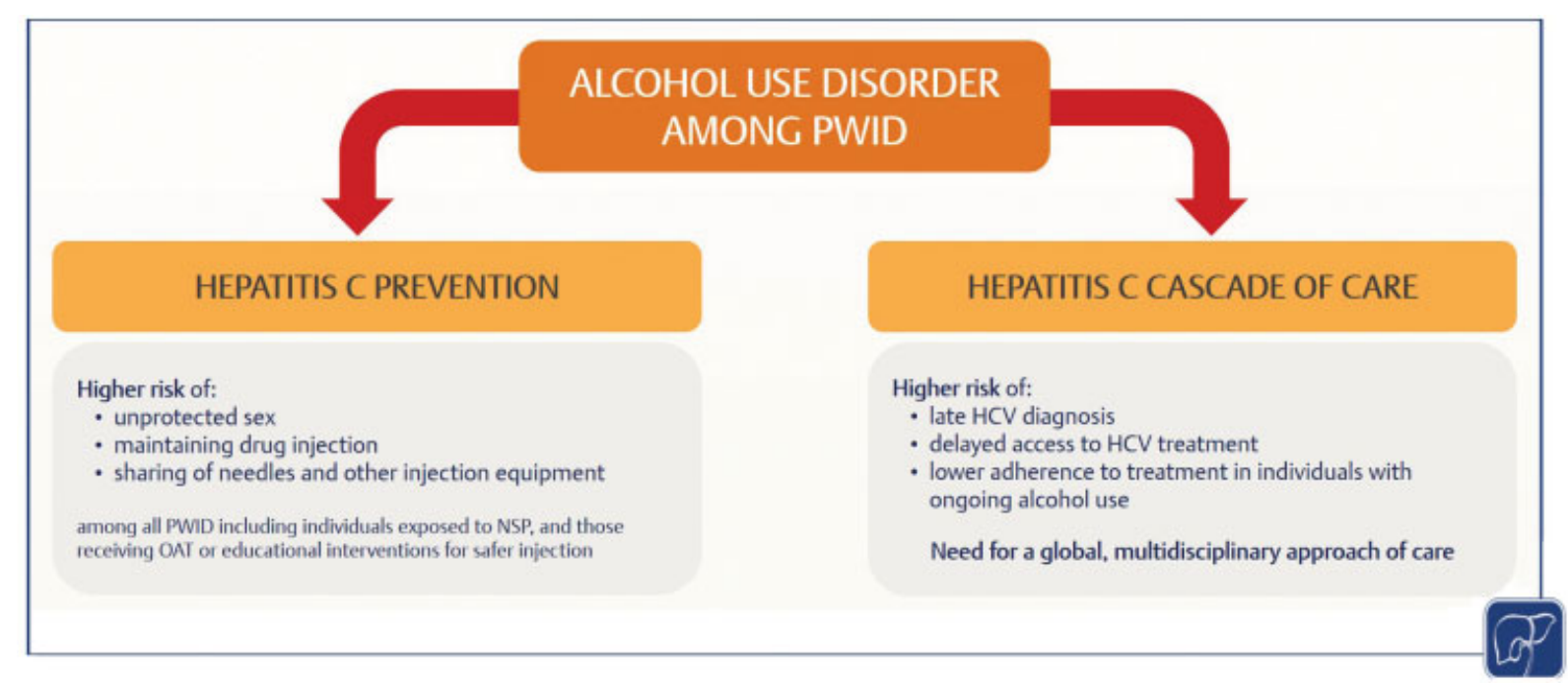

Fig. 1 Impact of AUD on HCV prevention and cascade of care. AUD, alcohol use disorder; HCV, hepatitis C virus; NSP, needle and syringe programs; OAT, opioid agonist therapy; PWID, people who inject drugs.

Another U.S. study conducted among 515 women receiving methadone maintenance treatment showed that those diagnosed with alcohol abuse or dependence disorder (according to the DSM-IV classification) had a more than twofold risk of unprotected sexual encounters and an 11-fold risk of having anal sex compared with other women. ${ }^{35}$ Similarly, a study conducted among 1,253 HIV-infected PWID entering detoxification or methadone maintenance treatment in New York City showed that drinking more than 14 alcohol drinks per week for males or seven alcohol drinks per week for females was associated with higher rates of injecting and sexual risk behaviors. ${ }^{36}$

In France, the community-based AERLI (Accompanying [support] and Educating to the Risk Linked to Injection) intervention provided face-to-face harm reduction educational sessions to PWID to decrease HIV- and HCV-related risk practices and other injecting-related complications. ${ }^{37}$ More than half of the 271 study participants reported harmful alcohol consumption (AUDIT-C [Alcohol Use Disorder Identification Test - Consumption] score ${ }^{38} \geq 3$ for women and $\geq 4$ for men) at enrolment, which was associated with an increased risk of unsafe HIV-HCV transmission practices. ${ }^{37}$ However, results showed no significant association between harmful alcohol consumption and HCV testing uptake. ${ }^{39}$

\section{Effect of AUD on HCV Treatment Uptake}

AUD has been associated with advanced liver disease stage (i.e., compensated cirrhosis or end-stage liver disease) at HCV diagnosis. For instance, alcohol abuse was the strongest predictor of advanced liver disease at initial HCV diagnosis in three U.S. Medicare cohorts. ${ }^{40}$

Until the early years of DAA availability, AUD remained a barrier to HCV treatment uptake or a reason for deferring HCV treatment initiation. ${ }^{41}$ This was partly due to some health care providers' concerns about heavy drinkers' ability to adhere to treatment. According to current guidelines, HCV-infected persons should be considered for HCV therapy regardless of alcohol intake. ${ }^{11,42}$ However, the European Association for the Study of the Liver (EASL) recommends additional support for individuals who consume alcohol while on HCV treatment. ${ }^{42}$

Research evidence has also supported the inclusion of alcohol users in DAA treatment. For example, a U.S. study conducted among 17,487 HCV-infected patients treated with DAA within the national Veterans Affairs health care system showed high sustained virologic response rates among all groups of patients regardless of alcohol use (assessed using the AUDIT-C). ${ }^{43}$

\section{Efficacy of Interventions to Manage AUD in People at Risk of HCV}

Pharmacotherapy for AUD remains globally underused in primary care, whereas medications such as acamprosate and oral naltrexone have been associated with a reduction in returning to drinking. ${ }^{44}$ The methodological heterogeneity across studies comparing psychosocial interventions for AUD in populations at greater risk of $\mathrm{HCV}$, such as PWID, makes it difficult to draw firm conclusions concerning their overall efficacy. ${ }^{45}$ However, some important points can be raised. First, alcohol reduction interventions may help decrease the level of consumption in HCV-infected individuals with moderate-to-high alcohol consumption. ${ }^{46}$ Second, brief motivational interventions can help reduce the frequency of injection-related blood-borne virus risk behaviors, as shown in hazardous drinkers in one needle exchange program. ${ }^{47}$ However, a systematic review ${ }^{45}$ did not find sufficient evidence supporting the effectiveness of brief interventions in reducing alcohol use in people with concurrent illicit drug use and AUD. Third, psychosocial therapy and participation in self-help groups are useful for patients with AUD affected by liver cirrhosis and/or hepatocellular carcinoma awaiting liver transplantation, even in the posttransplant period. ${ }^{48}$ Fourth, integrating harm reduction interventions for AUD in a more global health care setting for PWID, including social and support services (e.g., 
housing, shelter, welfare services) and other health services (such as dental care, mental health services, sexual and reproductive health services, etc.), has the potential to improve both access to and efficacy of these interventions, especially if PWID themselves are engaged in developing the services. $^{49}$

\section{Suggested Recommendations}

On the basis of this literature review, we propose the following recommendations to help reduce the impact of AUD on hepatitis C prevention and care among PWID.

\section{Standardizing Alcohol Use Assessment to Facilitate Research on AUD and HCV}

A variety of methods to assess alcohol consumption are used within the literature addressing the impact of AUD on HCV prevention and care, which makes it difficult to compare study results. In addition, the impact of alcohol use is often analyzed jointly with that of drug use, thus preventing the possibility to estimate the effects of each behavior independently. Furthermore, despite the common use of standard questionnaires in the literature on alcohol and HCV, a substantial proportion of published studies exploring this issue exclusively rely on post hoc measures of AUD from incomplete behavioral data (e.g., only asking participants about alcohol use during a given period of time).

An accurate measure of alcohol intake in a given setting requires a minimum knowledge of the types of alcoholic drinks regularly consumed and the ability to convert quantities into standard alcohol units. It is also paramount to guarantee the reproducibility of alcohol intake measures across various settings and populations to produce reliable comparisons.

As such, AUD impact studies require utilization of standardized measures of alcohol consumption or, at a minimum, systematic documentation of the number of alcohol units consumed. The diffusion and promotion of standard assessment tools, such as the AUDIT ${ }^{50-52}$ and the AUDIT$C_{,}^{38,51,52}$ constitute a key step in the documentation of alcohol use.

Moreover, alcohol use should be discussed with PWID, and more generally with all individuals infected with HCV or at risk of HCV infection, in a nonjudgmental manner to minimize social desirability bias in self-reported measures. Social desirability bias may be more present in countries where issues related to the use of alcohol and psychoactive substances are specifically difficult to investigate, as both people with AUD and PWID remain hidden populations. For instance, this is still the case in many parts of sub-Saharan Africa despite a strong heterogeneity between countries due to economic factors as well as factors related to religion, ethnicity, and availability and acceptability of alcohol in society $^{53}$ (-Table 2 ). In this context, the use of inexpensive and reliable point-of-care tests to cross-validate selfreported measurements of alcohol intake might improve reliability in alcohol use estimates. ${ }^{54}$
Table 2 Alcohol use in resource-limited settings: the case of Sub-Saharan Africa ${ }^{53}$

\begin{tabular}{|l|}
\hline Emerging alcohol market \\
\hline Widespread use of home-produced alcoholic beverages \\
\hline $\begin{array}{l}\text { AUD-related disease burden } \\
\text { resource settings }\end{array}$ \\
\hline $\begin{array}{l}\text { Hidden populations including people who inject drugs and } \\
\text { people with AUD }\end{array}$ \\
\hline $\begin{array}{l}\text { High abstention rates, with a strong heterogeneity between } \\
\text { countries due to economic factors, religion, ethnicity, and } \\
\text { availability and acceptability of alcohol in society }\end{array}$ \\
\hline
\end{tabular}

Abbreviation: AUD, alcohol use disorder.

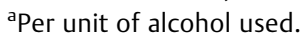

\section{Developing Harm Reduction Interventions}

Interventions aimed at reducing $\mathrm{HCV}$ transmission among PWID or other at-risk populations should target individuals who drink alcohol frequently and in high volumes as well as include strategies to reduce risky behaviors. Such harm reduction interventions are also needed to protect them from brain and liver injury. They must be adapted to the given cultural context, especially in populations for whom alcohol use is prohibited for religious or cultural reasons.

A recent review of $\mathrm{HCV}$ prevention/harm reduction interventions among PWID in 179 countries highlighted the need for greater investment in interventions as it showed that the coverage of these interventions (including OAT, NSP, and condom programs) remains insufficient to prevent, halt, and reverse the HCV epidemic among PWID. ${ }^{16}$ Furthermore, specific subgroups of PWID, such as methamphetamine injectors, may be at higher risk of insufficient NSP coverage and need care tailored to their distinct needs. ${ }^{55}$ Additionally, combining harm reduction interventions with social support interventions, such as stable housing, strengthens the effect of NSP on HCV prevention. ${ }^{56}$

\section{Scaling Up HCV Testing}

HCV testing remains largely insufficient among PWID. Expanding the use of point-of-care rapid tests in harm reduction and outreach services can greatly improve access to HCV care in this population, ${ }^{15}$ who are still highly discriminated against through, for example, repressive laws in many countries. Testing for HCV, especially if there is a positive diagnosis, can also be an important opportunity to assess for AUD and identify those in need of care.

\section{Engaging HCV At-Risk Populations in Treatment Despite Alcohol Use}

Modeling studies show that increasing access to DAA treatment in at-risk populations, such as PWID, will substantially impact the prevention of HCV transmission. ${ }^{10}$ However, HCV treatment coverage remains low in most 
at-risk populations, especially in eastern European countries, such as Russia. ${ }^{57}$ Expanding access to HCV treatment even among marginalized PWID will have both public health and social benefits. ${ }^{9,58}$ Engaging primary care providers/general practitioners in the screening, follow-up, and treatment of HCV infection can extend access to HCV care. ${ }^{59}$ The coordination of primary health care with addiction and HCV specialists is important to both engage and retain patients with AUD in HCV care. Evidence-based approaches of care delivery based on equity, nondiscrimination, and community engagement are needed for marginalized populations, such as PWID. Extending the use of practical tools such as service design checklists may help health policy-makers, caregivers, nongovernmental organizations, and communities to develop, update, or monitor care services for at-risk groups. ${ }^{49}$

\section{Conclusion}

To achieve the WHO goal of eliminating HCV as a public health threat, interventions must address alcohol misuse in at-risk populations, especially PWID. To adequately address AUD, policy-makers and care providers must prioritize providing more information on treatment and education to reduce alcohol-related harms, promoting an expanded range of treatment options, which incorporate brief interventions, and guaranteeing adequate case management. This spectrum of interventions should be proposed even to those still engaged in alcohol use. Furthermore, interventions to reduce AUD and prevent related harms in all individuals at risk of or living with HCV must be included in future research and policy agendas.

\section{Main Concepts and Learning Points}

- Alcohol use disorder (AUD) negatively impacts prevention, access to, and continuity of care for hepatitis $C$ virus (HCV) in at-risk populations, such as people who inject drugs (PWID).

- Specific interventions are required to reduce AUD and prevent related harms among PWID.

- Scaling up HCV testing and treating HCV-positive individuals despite AUD are needed to achieve micro-elimination in this key population.

- Research studies assessing AUD must use standardized measures of alcohol use to facilitate comparisons among studies.

- These measures must document, at a minimum, the number of alcohol units consumed in a given period of time.

\section{Author Contributions}

F. M. and P. C. drafted the manuscript. A. J., P. M., and J.V.L., contributed to the full manuscript and provided critical reviewing and suggestions throughout the different versions of this manuscript. All authors revised the manuscript and approved the final draft for submission.

\section{Funding}

F.M. and P.C. were supported by the French National Agency for Research on AIDS and Viral Hepatitis (Agence Nationale de Recherches sur le Sida et les hépatites virales, ANRS) and the French National Institute of Health and Medical Research (Institut National de la Santé et de la Recherche Médicale, INSERM). A.J. was supported by a grant from the National Cancer Institute, the Eunice Kennedy Shriver National Institute of Child Health \& Human Development, the National Institute of Allergy and Infectious Diseases, and the National Institute of Drug Abuse (grant no. 5U01AI069919). P.M. is supported by the National Institute of Health (NIH)/National Institute of Alcohol Abuse and Alcoholism (P60 AA009803). J.V.L. is supported by a Spanish Ministry of Science, Innovation, and Universities Miguel Servet grant (Instituto de Salud Carlos III/ESF, European Union [CP18/00074]) and further acknowledges support to ISGlobal from the Spanish Ministry of Science, Innovation, and Universities through the “Centro de Excelencia Severo Ochoa 2019-2023" Program (CEX2018-000806-S) and from the Government of Catalonia through the CERCA Program.

\section{Conflict of Interests}

The authors have no conflict of interest to declare with regard to this work.

\section{References}

1 American Psychiatric Association. Diagnostic and Statistical Manual of Mental Disorders (DSM-5). 5th ed. Washington, DC: American Psychiatric Association; 2013

2 GBD 2016 Alcohol and Drug Use Collaborators. The global burden of disease attributable to alcohol and drug use in 195 countries and territories, 1990-2016: a systematic analysis for the Global Burden of Disease Study 2016. Lancet Psychiatry 2018;5(12):987-1012

3 Alavi M, Janjua NZ, Chong M, et al. . The contribution of alcohol use disorder to decompensated cirrhosis among people with hepatitis C: an international study. J Hepatol 2018;68(03):393-401

4 Schwarzinger M, Baillot S, Yazdanpanah Y, Rehm J, Mallet V. Contribution of alcohol use disorders on the burden of chronic hepatitis C in France, 2008-2013: a nationwide retrospective cohort study. J Hepatol 2017;67(03):454-461

5 Fuster D, Sanvisens A, Bolao F, Rivas I, Tor J, Muga R. Alcohol use disorder and its impact on chronic hepatitis $C$ virus and human immunodeficiency virus infections. World J Hepatol 2016;8(31):1295-1308

6 Larney S, Randall D, Gibson A, Degenhardt L. The contributions of viral hepatitis and alcohol to liver-related deaths in opioiddependent people. Drug Alcohol Depend 2013;131(03):252-257

7 Ward JW, Hinman AR. What is needed to eliminate hepatitis B virus and hepatitis $C$ virus as global health threats. Gastroenterology 2019;156(02):297-310

8 World Health Organization. Access to hepatitis C testing and treatment for people who inject drugs and people in prisons - a global perspective: policy brief. Available at: https://www.who. int/hepatitis/publications/idu-prison-access-hepatitis-c/en/. Accessed May 27, 2020

9 Doyle JS, Aspinall EJ, Hutchinson SJ, et al. . Global policy and access to new hepatitis $C$ therapies for people who inject drugs. Int J Drug Policy 2015;26(11):1064-1071

10 Martin NK, Vickerman P, Grebely J, et al. . Hepatitis C virus treatment for prevention among people who inject drugs: modeling treatment scale-up in the age of direct-acting antivirals. Hepatology 2013;58(05):1598-1609 
11 American Association for the Study of Liver Diseases. Guidance HCV: Recommendations for Testing, Managing, and Treating Hepatitis C. Available at: https://www.hcvguidelines.org/. Accessed May 27, 2020

12 Razavi H, Sanchez Gonzalez Y, Yuen C, Cornberg M. Global timing of hepatitis $\mathrm{C}$ virus elimination in high-income countries. Liver Int 2020;40(03):522-529

13 Dore GJ, Martinello M, Alavi M, Grebely J. Global elimination of hepatitis C virus by 2030: why not? Nat Med 2020;26(02): 157-160

14 Lazarus JV, Safreed-Harmon K, Thursz MR, et al. . The microelimination approach to eliminating hepatitis C: strategic and operational considerations. Semin Liver Dis 2018;38(03): 181-192

15 Lazarus JV, Pericàs JM, Colombo M, Ninburg M, Wiktor S, Thursz M. Viral hepatitis: "E" is for equitable elimination. J Hepatol 2018; 69(04):762-764

16 Larney S, Peacock A, Leung J, et al. . Global, regional, and countrylevel coverage of interventions to prevent and manage HIV and hepatitis $C$ among people who inject drugs: a systematic review. Lancet Glob Health 2017;5(12):e1208-e1220

17 Smith DJ, Combellick J, Jordan AE, Hagan H. Hepatitis C virus (HCV) disease progression in people who inject drugs (PWID): a systematic review and meta-analysis. Int J Drug Policy 2015;26 (10):911-921

18 Safreed-Harmon K, Blach S, Aleman S, et al. . The consensus hepatitis $C$ cascade of care: standardized reporting to monitor progress toward elimination. Clin Infect Dis 2019;69(12): 2218-2227

19 Siu L, Foont J, Wands JR. Hepatitis C virus and alcohol. Semin Liver Dis 2009;29(02):188-199

20 Meyer JP, Isaacs K, El-Shahawy O, Burlew AK, Wechsberg W. Research on women with substance use disorders: reviewing progress and developing a research and implementation roadmap. Drug Alcohol Depend 2019;197:158-163

21 Dillon JF, Lazarus JV, Razavi HA. Urgent action to fight hepatitis C in people who inject drugs in Europe. Hepatol Med Policy 2016;1:2

22 Degenhardt L, Peacock A, Colledge S, et al. . Global prevalence of injecting drug use and sociodemographic characteristics and prevalence of HIV, HBV, and HCV in people who inject drugs: a multistage systematic review. Lancet Glob Health 2017;5(12): e1192-e1207

23 Grebely J, Tran L, Degenhardt L, et al. . Association between opioid agonist therapy and testing, treatment uptake, and treatment outcomes for hepatitis $C$ infection among people who inject drugs: a systematic review and meta-analysis. Clin Infect Dis Off Publ Infect Dis Soc Am 2020 (e-pub ahead of print) . Doi: $10.1093 /$ cid/ciaa612

24 World Health Organization. WHO, UNODC, UNAIDS: technical guide for countries to set targets for universal access to HIV prevention, treatment and care for injecting drug users. Available at: https://www.unaids.org/sites/default/files/sub_landing/idu_target_setting_guide_en.pdf. Accessed May 27, 2020

25 Platt L, Minozzi S, Reed J, et al. . Needle and syringe programmes and opioid substitution therapy for preventing HCV transmission among people who inject drugs: findings from a Cochrane Review and meta-analysis. Addiction 2018;113(03):545-563

26 Welch-Lazoritz M, Hautala D, Habecker P, Dombrowski K. Association between alcohol consumption and injection and sexual risk behaviors among people who inject drugs in rural Puerto Rico. J Subst Abuse Treat 2017;82:34-40

27 Poudel KC, Poudel-Tandukar K, Yasuoka J, Joshi AB, Jimba M. Correlates of sharing injection equipment among male injecting drug users in Kathmandu, Nepal. Int J Drug Policy 2010;21(06): 507-510

28 Stein MD, Hanna L, Natarajan R, et al. . Alcohol use patterns predict high-risk HIV behaviors among active injection drug users. J Subst Abuse Treat 2000;18(04):359-363
29 Roux P, Fugon L, Jones JD, Comer SD. Hepatitis C infection in nontreatment-seeking heroin users: the burden of cocaine injection. Am J Addict 2013;22(06):613-618

30 Evans JL, Hahn JA, Lum PJ, Stein ES, Page K. Predictors of injection drug use cessation and relapse in a prospective cohort of young injection drug users in San Francisco, CA (UFO Study). Drug Alcohol Depend 2009;101(03):152-157

31 Gyarmathy VA, Neaigus A. The association between social marginalisation and the injecting of alcohol amongst IDUs in Budapest, Hungary. Int J Drug Policy 2011;22(05):393-397

32 Mehta SH, Srikrishnan AK, Noble E, et al. . Emergence of cocaine and methamphetamine injection among HIV-positive injection drug users in northern and western India. Drug Alcohol Depend 2014:135:160-165

33 Hillebrand J, Marsden J, Finch E, Strang J. Excessive alcohol consumption and drinking expectations among clients in methadone maintenance. J Subst Abuse Treat 2001;21(03):155-160

34 Chaudhry AA, Botsko M, Weiss L, et al; BHIVES Collaborative. Participant characteristics and HIV risk behaviors among individuals entering integrated buprenorphine/naloxone and HIV care. J Acquir Immune Defic Syndr 2011;56(Suppl 1):S14-S21

35 Tross S, Hanner J, Hu MC, Pavlicova M, Campbell A, Nunes EV. Substance use and high risk sexual behaviors among women in psychosocial outpatient and methadone maintenance treatment programs. Am J Drug Alcohol Abuse 2009;35(05):368-374

36 Arasteh K, Des Jarlais DC. At-risk drinking and injection and sexual risk behaviors of HIV-positive injection drug users entering drug treatment in New York City. AIDS Patient Care STDS 2009;23(08): 657-661

37 Roux P, Le Gall JM, Debrus M, et al. . Innovative community-based educational face-to-face intervention to reduce HIV, HCV and other blood-borne infectious risks in difficult-to-reach people who inject drugs: results from the ANRS-AERLI intervention study. Addiction 2016;111(01):94-106

38 Bush K, Kivlahan DR, McDonell MB, Fihn SD, Bradley KA. The AUDIT alcohol consumption questions (AUDIT-C): an effective brief screening test for problem drinking. Ambulatory Care Quality Improvement Project (ACQUIP). Alcohol Use Disorders Identification Test. Arch Intern Med 1998;158(16):1789-1795

39 Roux P, Rojas Castro D, Ndiaye K, et al. . Increased uptake of HCV testing through a community-based educational intervention in difficult-to-reach people who inject drugs: results from the ANRS-AERLI study. PLoS One 2016;11(06):e0157062

40 Chirikov VV, Shaya FT, Howell CD. Contextual analysis of determinants of late diagnosis of hepatitis C virus infection in Medicare patients. Hepatology 2015;62(01):68-78

41 Papatheodoridis GV, Tsochatzis E, Hardtke S, Wedemeyer H. Barriers to care and treatment for patients with chronic viral hepatitis in Europe: a systematic review. Liver Int 2014;34(10):1452-1463

42 European Association for the Study of the Liver. Electronic address: easloffice@easloffice.eu; European Association for the Study of the Liver. EASL recommendations on treatment of hepatitis C 2018. J Hepatol 2018;69(02):461-511

43 Tsui JI, Williams EC, Green PK, Berry K, Su F, Ioannou GN. Alcohol use and hepatitis $C$ virus treatment outcomes among patients receiving direct antiviral agents. Drug Alcohol Depend 2016;169:101-109

44 Jonas DE, Amick HR, Feltner C, et al. . Pharmacotherapy for Adults With Alcohol-Use Disorders in Outpatient Settings. Rockville, MD: Agency for Healthcare Research and Quality; 2014

45 Klimas J, Tobin H, Field C-A, et al. . Psychosocial interventions to reduce alcohol consumption in concurrent problem alcohol and illicit drug users. Cochrane Database Syst Rev 2014;(12):CD009269pub3

46 Doyle JS, Hunt D, Aspinall EJ, et al. . A systematic review of interventions to reduce alcohol consumption among individuals with chronic HCV infection. J Hepatol 2014;60(01):S314-S315

47 Stein MD, Anderson B, Charuvastra A, Maksad J, Friedmann PD. A brief intervention for hazardous drinkers in a needle exchange program. J Subst Abuse Treat 2002;22(01):23-31 
48 Testino G, Leone S, Borro P. Treatment of alcohol use disorder patients affected by liver cirrhosis and/or hepatocellular carcinoma awaiting liver transplantation. Minerva Med 2016;107(04):223-238

49 Lazarus JV, Baker L, Cascio M, et al; Nobody Left Outside initiative. Novel health systems service design checklist to improve healthcare access for marginalised, underserved communities in Europe. BMJ Open 2020;10(04):e035621

50 World Health Organization. The Alcohol Use Disorders Identification Test - Guidelines for use in primary care. 2nd ed. Available at: https://apps.who.int/iris/handle/10665/67205. Accessed May 27, 2020

51 Pilowsky DJ, Wu LT. Screening for alcohol and drug use disorders among adults in primary care: a review. Subst Abuse Rehabil 2012;3(01):25-34

52 Jin J. Screening and counseling to reduce unhealthy alcohol use. JAMA 2018;320(18):1948

53 De Bruijn A. Alcohol marketing practices in Africa - Findings from the Gambia, Ghana, Nigeria and Uganda - MAMPA Project. Available at: http://eucam.info/wp-content/uploads/2014/04/ mampa-2010-report-findings_final-version-22-june-2011.pdf. Accessed May 27, 2020

54 Vinikoor MJ, Zyambo Z, Muyoyeta M, Chander G, Saag MS, Cropsey K. Point-of-care urine ethyl glucuronide testing to detect alcohol use among HIV-hepatitis B virus coinfected adults in Zambia. AIDS Behav 2018;22(07):2334-2339

55 O'Keefe D, Scott N, Aitken C, Dietze P. Longitudinal analysis of change in individual-level needle and syringe coverage amongst a cohort of people who inject drugs in Melbourne, Australia. Drug Alcohol Depend 2017;176:7-13

56 Gibson EK, Exner H, Stone R, Lindquist J, Cowen L, Roth EA. A mixed methods approach to delineating and understanding injection practices among clientele of a Victoria, British Columbia needle exchange program. Drug Alcohol Rev 2011;30(04): 360-365

57 Tsui JI, Ko SC, Krupitsky E, et al. . Insights on the Russian HCV care cascade: minimal HCV treatment for HIV/HCV co-infected PWID in St. Petersburg. Hepatol Med Policy 2016;1:13

58 Read P, Lothian R, Chronister K, et al. . Delivering direct acting antiviral therapy for hepatitis $\mathrm{C}$ to highly marginalised and current drug injecting populations in a targeted primary health care setting. Int J Drug Policy 2017;47:209-215

59 Woodrell C, Weiss J, Branch A, et al. . Primary care-based hepatitis $\mathrm{C}$ treatment outcomes with first-generation direct-acting agents. J Addict Med 2015;9(05):405-410

60 Bazzi A, Saitz R. Screening for unhealthy alcohol use. JAMA 2018; 320(18):1869-1871

61 US Department of Health and Human Services, National Institutes of Health, National Institute on Alcohol Abuse and Alcoholism. Helping Patients Who Drink Too much: A Clinician's Guide. Updated 2005 Edition. Bethesda, MD: National Institutes of Health; 2007

62 World Health Organization. Guidelines for the care and treatment of persons diagnosed with chronic hepatitis $C$ virus infection. Available at: https://www.who.int/hepatitis/publications/hepatitis-c-guidelines-2018/en/. Accessed May 27, 2020

63 Reus VI, Fochtmann LJ, Bukstein O, et al. . The American Psychiatric Association Practice guideline for the pharmacological treatment of patients with alcohol use disorder. Am J Psychiatry 2018; 175(01):86-90

64 O'Connor EA, Perdue LA, Senger CA, et al. . Screening and behavioral counseling interventions to reduce unhealthy alcohol use in adolescents and adults: updated evidence report and systematic review for the US Preventive Services Task Force. JAMA 2018;320(18):1910-1928 